Pacific Journal of Mathematics

ON FINITE GROUPS WITH INDEPENDENT CYCLIC SYLOW 


\title{
ON FINITE GROUPS WITH INDEPENDENT CYCLIC SYLOW SUBGROUPS
}

\author{
MARCEL HERZOG
}

The purpose of this paper is to classify all perfect groups with cyclic Sylow $p$-subgroups which satisfy the condition

(TI) two different Sylow $p$-subgroups of $G$ contain only and such that the unit element in common

$$
o(G)<o(P)^{3}
$$

where $P$ is a Sylow $p$-subgroup of $G$.

The main result of this paper is the following

Theorem 1. Let $G$ be a perfect finite group with a cyclic Sylow $p$-subgroup $P$ of order $p^{a}$ and assume that the Sylow $p$-subgroups of $G$ satisfy the $(T I)$ condition. Assume, furthermore, that

$$
o(G)<p^{3 a} .
$$

Then one of the following statements holds.

(I) $a=1, G \cong P S L(2, p)$, where $p>3$ is a prime.

(II) $a=1, G \cong P S L(2, p-1)$, where $p=2^{m}+1>5$ is a Fermat prime.

(III) $a=1, G \cong S L(2, p)$, where $p>3$ is a prime.

(IV) $a=2, p=3, G \cong P S L(2,8)$.

Ten years ago E. Artin raised the following problem: what are the simple finite groups $G$ of order $g$ which are divisible by a prime $p>g^{1 / 3}$ ? This question was answered by R. Brauer and W. F. Reynolds in [1]. They found that the only groups satisfying the above conditions are $P S L(2, p)$, where $p>3$ is a prime, and $P S L(2$, $p-1$ ) where $p>3$ is a Fermat prime, $p=2^{m}+1$. In particular, the Sylow $p$-subgroups of these groups are of order $p$ and therefore they are cyclic and satisfy the $(T I)$ condition. Theorem 1 thus generalizes these results.

As a matter of fact we will prove a more general statement than Theorem 1.

Theorem 1*. Let $G$ be a finite group with a cyclic Sylow psubgroup $P$ of order $p^{a}$ and assume that the Sylow p-subgroups of $G$ satisfy the (TI) condition. Assume, furthermore, that

$$
o(G)<p^{3 a}
$$

and no homomorphic image of $G$ is isomorphic to $N_{G}(P) / W$, where 
$W$ is the normal complement of $P$ in $C_{G}(P)$. Then one of the following statements holds.

(I )* $a=1, G \cong P S L(2, p)$, where $p>3$ is a prime.

(II )* $a=1, \quad G \cong P S L(2, p-1)$, where $p=2^{m}+1>5$ is a Fermat prime.

(III)* $\quad a=1, G \cong S L(2, p)$, where $p>3$ is a prime.

$(\mathrm{IV})^{*} \quad a=2, p=3, G \cong P S L(2,8)$.

$(\mathrm{V})^{*} \quad a=1, G \cong P G L(2, p)$, where $p>3$ is a prime.

$(\mathrm{VI})^{*} \quad a=1, G \cong P S L(2, p) \times M$, where $p>3$ is a prime and $o(M)=2$.

Since $G=G^{\prime}$ implies the last condition of Theorem $1^{*}$, Theorem 1 follows immediately from Theorem $1^{*}$. In this paper the group $N_{G}(P) / W$ will be referred to as the $p$-metacyclic group of order $q p^{a}$.

Theorem $1^{*}$ follows from the following more general result:

Theorem 2. Let $G$ be a finite group with a cyclic Sylow p-subgroup $P$ of order $p^{a}>1$ and assume that the Sylow p-subgroups of $G$ satisfy the (TI) condition. Suppose that no homomorphic image of $G$ is isomorphic to the p-metacyclic group of order $p^{a} q$. Then

$$
o(G)=q w p^{a}\left(1+n p^{a}\right)
$$

where $w p^{a}=o\left(C_{G}(P)\right), q=\left[N_{G}(P): C_{G}(P)\right]>1$ and $n$ is a positive integer.

Furthermore, let $G_{0}$ be the minimal normal subgroup of $G$ for which $G / G_{0}$ is solvable, and let $M$ be the maximal normal subgroup of $G_{0}$ of order prime to $p$. Denote $G_{0} / M$ by $G^{*}$. Then one of the following statements holds.

(A) $n=\left(h v p^{a}+h+v^{2}+v\right) /(v+1)$

where $h$ and $v$ are positive integers and $v+1 \mid h\left(p^{a}-1\right)$.

(B) $a=1, n=1, G^{*} \cong P S L(2, p)$ where $p>3$ is a prime.

(C) $a=1, n=(p-3) / 2, G^{*} \cong P S L(2, p-1)$ where $p=2^{m}+1>5$ is a Fermat prime.

(D) $a=2, p=3, n=\left(p^{2}-3\right) / 2, G^{*} \cong \operatorname{PSL}(2,8)$.

Theorem 2 immediately yields

COROLlary. Let $G$ satisfy the assumptions of Theorem 2 and suppose that $n<\left(p^{a}+3\right) / 2$. Then $G^{*}$ is of type (B), (C) or (D).

In $\S 2$ some basic properties of groups with a Sylow subgroup satisfying the $T I$-property are derived. Section 3 contains the proof of Theorem 2, from which Theorem $1^{*}$ is deduced in $\S 4$.

We use the standard notation $C_{G}(T), N_{G}(T), o(T), T^{\sharp}$, and $\langle T\rangle$, 
where $T$ is a subset of the group $G$, to denote respectively: the centralizer, normalizer, number of elements, the nonunit elements and the group generated by $T$. We will say that $N_{G}(T) / C_{G}(T)$ acts frobeniusly on $T$ if $\theta^{\eta}=\theta$ for $\theta \in T^{\#}$ and $\eta \in N_{G}(T)$ implies that $\eta \in C_{G}(T)$. An element of $G$ is called a $p^{\prime}$-element, where $p$ is a prime number, if $p$ does not divide its order. The principal character and the commutator subgroup of $G$ will be denoted by $1_{G}$ and $G^{\prime}$ respectively. Finally, if $a$ and $b$ are integers, then $(a, b)$ denotes their greatest common divisor and $a \mid b$ means: $a$ divides $b$.

2. TIP-groups. A finite group will be called a TIP-group if its Sylow $p$-subgroups are nontrivial and satisfy the $T I$-property. This section deals with properties of $T I P$-groups in general, followed by a study of TIP-groups with a cyclic Sylow $p$-subgroup.

Proposition 2.1. Let $G$ be a TIP-group with a Sylow p-subgroup $P$ of order $p^{a}$. Then the following statements hold.

(a) $C_{G}(P)=W \times P$ where $o(W)=w$ and $(w, p)=1$.

( b ) $o(G)=q w p^{a}\left(1+n p^{a}\right)$ where $q=\left[N_{G}(P): C_{G}(P)\right]$ and $n$ is a nonnegative integer.

(c) Any normal subgroup $L$ of $G$ of order divisible exactly by $p^{b}>1$ is a TIP-group of order $q_{L} w_{L} p^{b}\left(1+n p^{a}\right)$.

(d) If $H$ is a normal subgroups of $G$ of order prime to $p$, then $G / H$ is a TIP-group.

Proof. Let $C=C_{G}(P), N=N_{G}(P)$.

(a) Since $P$ is a normal Hall-subgroup of $C$, it has a complement $W$ and $(w, p)=1$. Since elements of $W$ commute with elements of $P, C=W \times P$.

(b) Consider the conjugates $\left\{P_{i}\right\}$ of $P$, other than $P$. If $\sigma \in P$ and $P_{i}^{\sigma}=P_{i}$, where $P_{i}=P^{\tau}, \tau \in G$, then $P^{\tau \sigma \tau-1}=P, \tau \sigma \tau^{-1} \in N_{G}(P)$ and $\sigma \in N_{G}\left(P^{\tau}\right), \sigma \in P \cap P^{\tau}=\{1\}$. Thus $P$ acts by conjugation fixed point free on $\left\{P_{i}\right\}$ and therefore $o\left\{P_{i}\right\}=n p^{a}$ for some nonnegative integer $n$. Hence $[G: N]=1+n p^{a}$ and $o(G)=q w p^{a}\left(1+n p^{a}\right)$.

(c) - (d) The proof of Lemma 1 in [6] obviously holds also for general TIP-groups, with $p \neq 2$. Thus any subgroup of $G$ of order divisible by $p$ is a TIP-group and (d) holds. Let $o(L)=q_{L} x_{L} p^{b}\left(1+n_{L} p^{b}\right)$. Since $L$ and $G$ have the same number of Sylow $p$-subgroups $1+n_{L} p^{b}=$ $1+n p^{a}$ proving (c).

Proposition 2.2. Let $G$ be a TIP-group with a cyclic Sylow p-subgroup $P$ of order $p^{a}$. Then in addition to properties (a)-(d) of Proposition 2.1, and using the same notation, the following state- 
ments hold.

(e) $C_{G}(P)=C_{G}(\sigma)$ and $N_{G}(P)=N_{G}(\langle\sigma\rangle)$

for all $\sigma \in P^{\sharp}$.

(f) $q$ divides $p-1$.

(g) $o(G / H)=q \bar{w} p^{a}\left(1+\bar{n} p^{a}\right)$

and there exists a nonnegative integer $z$ such that

$$
n=z+\bar{n}+z \bar{n} p^{a} .
$$

If $z=0$ then $H \subset W$.

(h) If $K$ is a normal subgroup of $G$ and $K$ does not contain $P$ then

$$
N_{K}(P)=C_{K}(P) \text {. }
$$

(i) If also $o(K \cap P)>1$, then $G$ can be mapped homomorphically on the p-metacyclic group of order $p^{a} q$.

Proof. (e ) Let $\sigma \in P^{\sharp}$; then by Lemma 2.1.b in [3] $C_{G}(\sigma) \cap N_{G}(P)=$ $C_{G}(P)$. It follows from the $T I$-property that $C_{G}(\sigma) \subset N_{G}(P)$ and $N_{G}(\langle\sigma\rangle) \subset N_{G}(P)$. Thus $C_{G}(\sigma)=C_{G}(P)$ and since $P$ is cyclic $N_{G}(\langle\sigma\rangle)=$ $N_{G}(P)$.

(f) By Lemma 2.1.d of [3] $N / C$ acts frobeniusly on $P$ and $P$ is cyclic. Therefore $q=[N: C]$ divides $p-1$.

(g) The proof of Proposition 2 in [1] holds, with the obvious changes, also in the present case. It is clear from the proof in [1] that if $z=0$ then $H \subset C_{G}(P)$.

(h) Suppose that $K \cap N \not \subset C$ and let $\sigma \in K \cap N-C$. Since $N / C$ acts frobeniusly on $P$, it follows that the elements $\sigma \rho^{-1} \sigma^{-1} \rho, \rho \in P$, are distinct and belong to $P \cap K$. Thus $P$ is contained in $K$, a contradiction. Consequently $K \cap N \subset C$ and $K \cap N=K \cap C$, as required.

(i) Let $p^{b}$ be the exact power of $p$ dividing $o(K)$. Then $1<p^{b}<p^{a}$ and by Proposition 2.1.c and (h) $o(K)=w_{K} p^{b}\left(1+n p^{a}\right)$, where $w_{K} p^{b}=o\left(C_{K}(P \cap K)\right)=o\left(N_{K}(P \cap K)\right)$. By the Burnside Theorem $K$ has a characteristic subgroup $T$ of order $w_{K}\left(1+n p^{a}\right) . \quad T$ is normal in $G$ and $G=N T$. Consequently $W T$ is a normal subgroup of $G$ and $G / W T$ is isomorphic to the $p$-metacyclic group of order $p^{a} q$.

3. Proof of Theorem 2. If either $p=2$ or $q=1$, then $C_{G}(P)=N_{G}(P)$ and by the Burnside Theorem $P$ has a normal complement in $G$, in contradiction to our assumption. Thus $p>2$ and $q>1$.

If $P$ is normal in $G$ and $C_{G}(P)=W \times P$, then $W$ is normal in $G$, again a contradiction. Thus $P$ is not normal in $G$ and the first statement of Theorem 2 follows from Proposition 2.1.b.

It follows from Proposition 3 in [1] and Proposition 2.2.i that 
$P \subset G_{0}$. Indeed, if $P \not \subset G_{0}$ then either $a=1$ or $a>1$ and $G$ contains a normal subgroup $U$ such that $1<o(U \cap P)<p^{a}$. In both cases the above mentioned propositions yield a contradiction to our assumptions.

The definition of $G_{0}$ forces it to be its own commutator subgroup and the same is true for $G^{*}$. Moreover, $G^{*}$ does not have nontrivial normal subgroups of order prime to $p$.

From now on we will assume that (A) is not satisfied and will show that then one of the statements $(\mathrm{B}),(C)$, or $(D)$ holds.

Let $o\left(G_{0}\right)=q_{0} w_{0} p^{a}\left(1+n p^{a}\right), o\left(G^{*}\right)=q_{0} w^{*} p^{a}\left(1+n^{*} p^{a}\right)$. Since $G^{*}=$ $\left(G^{*}\right)^{\prime}, n^{*} \neq 0$. By Proposition 2.2.g there exists a nonnegative integer $z$ such that

$$
n=z+n^{*}+z n^{*} p^{a} .
$$

If $z \neq 0$, let $h=(z+1) n^{*}, v=z$. Then:

$$
n=v+h /(v+1)+v h p^{a} /(v+1)
$$

in contradiction to our assumptions. Thus $z=0$ and $n^{*}=n$.

Consequently, it suffices to show that if $G$ satisfies the assumptions of Theorem 2 and in addition, $G=G^{\prime}, G$ has no nontrivial normal subgroup of order prime to $p$ and $n$ does not satisfy (A), then $G$ is isomorphic to one of the simple groups described in (B), (C), and (D).

We will use the following notation: $N=N_{G}(P), C=C_{G}(P)=$ $W \times P$ where $o(W)=w$ and $(w, p)=1$.

Let $B$ be the principal $p$-block of $G$. Then by Proposition 2.1 of [3] $B$ contains $t=\left(p^{a}-1\right) / q$ exceptional characters $X_{\lambda}$ of degree $x_{0}$, $\lambda=1, \cdots, t$ and $q$ nonexceptional characters $X_{i}$ of degree $x_{i}, i=$ $1, \cdots, q$. If $\sigma \in P^{*}$ and $\pi$ is a $p^{\prime}$-element of $C_{G}(\sigma)=C$ then:

$$
\begin{array}{ll}
X_{\lambda}(\sigma \pi)=-\varepsilon_{0} \sum_{\rho \in R} \zeta_{\lambda}^{o}(\sigma) & \text { for } \lambda=1, \cdots, t \\
X_{j}(\sigma \pi)=\varepsilon_{j} & \text { for } j=1, \cdots, q
\end{array}
$$

where $R$ is a set of coset representatives of $C$ in $N,\left\{\zeta_{\lambda} \mid \lambda=1, \cdots, t\right\}$ is a set of representatives of the $t$ transitivity classes of characters of $P$ under conjugation by $N$ (see [3], Lemma 2.2), and $\varepsilon_{j}= \pm 1$ for $j=0,1, \cdots, q$. It follows also by Corollary 2.1 of [3] that the following relations hold:

$$
\begin{aligned}
x_{i} & \equiv \varepsilon_{i}\left(\bmod p^{a}\right) \\
t x_{0} & \equiv \varepsilon_{0}\left(\bmod p^{a}\right)
\end{aligned} \quad \text { for } i=1, \cdots, q
$$

and

$$
\sum_{i=0}^{q} \varepsilon_{i} x_{i}=0
$$


We are now ready to state

LEMMA 3.1.

$$
t x_{j} \mid\left(p^{a}-1\right)\left(1+n p^{a}\right) \quad \text { for } j=0, \cdots, q .
$$

Proof. If $\sigma \in P^{\sharp}$, then $C=C_{G}(\sigma)$ and it is well-known that the expression

$$
\frac{o(G) \cdot X_{j}(\sigma)}{o(C) \cdot x_{j}}
$$

is an algebraic integer for all $j$. It follows, from Proposition 2.1 and (1), that for $j=1, \cdots, q$

$$
q w p^{a}\left(1+n p^{a}\right) / w p^{a} x_{j}
$$

is an algebraic integer and consequently

$$
t x_{j} \mid t q\left(1+n p^{a}\right)=\left(p^{a}-1\right)\left(1+n p^{a}\right) .
$$

For $j=0$, it follows from (1), Proposition 2.1 and Lemma 2.2 of [3] that

$$
\sum_{\lambda=1}^{t} \frac{o(G) X_{\lambda}(\sigma)}{o(C) x_{0}}=\frac{q w p^{a}\left(1+n p^{a}\right) \varepsilon_{0}}{w p^{a} x_{0}}
$$

is an algebraic integer and therefore $t x_{0} \mid\left(p^{a}-1\right)\left(1+n p^{a}\right)$.

Since the block $B$ contains $1_{G}$ as a nonexceptional character, we may assume that $X_{1}=1_{G}$. We have then

Lemma 3.2. For $j=0,2,3, \cdots, q$

$$
\bar{x}_{j}=\left\{\begin{array}{lll}
1+n p^{a} & \text { if } & \varepsilon_{j}=1 \\
p^{a}-1 & \text { if } & \varepsilon_{j}=-1
\end{array}\right.
$$

where $\bar{x}_{j}=x_{j}$ for $j=2, \cdots, q$ and $\bar{x}_{0}=t x_{0}$.

Proof. We will show first that if

$$
u p^{a}+\varepsilon \mid\left(p^{a}-1\right)\left(1+n p^{a}\right), \quad \varepsilon= \pm 1
$$

then either $n$ satisfies statement (A) or one of the following relations holds:

$$
\begin{array}{lll}
u p^{a}+\varepsilon=1 & \text { or } \quad n p^{a}+1 & \text { if } \varepsilon=1 \\
u p^{a}+\varepsilon=p^{a}-1 & \text { or } \quad\left(p^{a}-1\right)\left(n p^{a}+1\right) & \text { if } \varepsilon=-1 .
\end{array}
$$

To do so, it suffices to show that if $n$ does not satisfy $(A)$ then the only solutions of 


$$
\left(v p^{a}+1\right)\left(w p^{a}-1\right)=\left(p^{a}-1\right)\left(1+n p^{a}\right)
$$

in nonnegative integers $v$ and $w$ are: $v=0, w p^{a}-1=\left(p^{a}-1\right)\left(1+n p^{a}\right)$ and $v=n, w=1$.

Suppose that $v \neq 0$ and $w>1$. Then $v p^{a}+1<1+n p^{a}, v<n$. By multiplying out equation (5), adding 1 to both sides and dividing by $p^{a}$ we get

$$
w v p^{a}+w-v=n p^{a}-n+1 .
$$

Now by (6):

$$
\begin{aligned}
\left(v p^{a}+1\right)(n-w v) & =v p^{a} n-v\left(w v p^{a}\right)+n-w v \\
& =v p^{a} n+v w-v^{2}-v n p^{a}+v n-v+n-w v \\
& =(n-v)(v+1) .
\end{aligned}
$$

Since $n>v$, the left hand side of the equation is positive and so we may put $h=n-w v$, where $h$ is a positive integer. Solving for $n$ we get a contradiction to the assumption that $n$ does not satisfy (A). Thus either $v=0$ or $w=1$ and the above assertion follows.

Now we have seen that for $j=0,2,3, \cdots, q$

$$
\bar{x}_{j} \equiv \varepsilon_{j}\left(\bmod p^{a}\right) \text { and } \bar{x}_{j} \mid\left(p^{a}-1\right)\left(1+n p^{a}\right) \text {. }
$$

Since $X_{1}$ is the only character of $G$ of degree 1 , it follows that for $j=0,2,3, \cdots, q$

$$
\bar{x}_{j}= \begin{cases}1+n p^{a} & \text { if } \quad \varepsilon_{j}=1 \\ p^{a}-1 \text { or } \quad\left(p^{a}-1\right)\left(1+n p^{a}\right) & \text { if } \quad \varepsilon_{j}=-1 .\end{cases}
$$

Thus it suffices to show that for $j=0,2,3, \cdots, q$

$$
\bar{x}_{j} \neq\left(p^{a}-1\right)\left(1+n p^{a}\right) .
$$

Indeed, if the equality holds, then by (3) :

$$
0=\sum_{i=0}^{q} \varepsilon_{i} x_{i} \leqq 1+(q-1)\left(1+n p^{a}\right)-\left(p^{a}-1\right)\left(1+n p^{a}\right) / t=-n p^{a}
$$

a contradiction. The proof of Lemma 3.2 is complete.

We will proceed with the proof of Theorem 2. It follows from (3) that at least one of the $\varepsilon_{j}^{\prime} \mathrm{s}, j=0,1, \cdots, q$, is negative. If $\varepsilon_{0}=$ -1 , let $X=\sum_{i=1}^{t} X_{\lambda}$ and if $\varepsilon_{i}=-1$ for some $i \geqq 2$ let $X=X_{i}$. In either case, by Lemma $3.2 X$ is a character of $G$ of degree $p^{a}-1$ and by (1) and Lemma 2.2 of [3]

$$
X(\sigma \pi)=-1
$$

for $\sigma \in P^{\sharp}, \pi \in W$, where $C=P \times W$. Denote the restriction of $X$ to 
$C$ also by $X$; then $X$ is a character of $P \times W$ and therefore for $\rho \in P$ and $\pi \in W$ we have:

$$
X(\rho \pi)=\sum_{i=1}^{r} \psi_{i}(\pi) \varphi_{i}(\rho)
$$

where $\psi_{i}, i=1, \cdots, r$ are distinct irreducible characters of $W$ and $\varphi_{i}, i=1, \cdots, r$ are characters of $P$. Let $\sigma \in P^{\sharp}, \pi \in W$; as $X(\sigma \pi)=$ -1 , it follows from (7) and from the linear independence of the irreducible characters of $W$, that the principal character appears among the $\psi_{i}$, say $\psi_{1}=1_{W}$, and

$$
\varphi_{1}(\sigma)=-1, \varphi_{2}(\sigma)=\cdots=\varphi_{r}(\sigma)=0 .
$$

Suppose that $r>1$. Then $\varphi_{2}$ vanishes on $P^{*}$ and therefore $p^{a}$ divides $\varphi_{2}(1)$, in contradiction to $(7)$ and the fact that $X(1)=p^{a}-1$. Thus $r=1$ and

$$
X(\rho \pi)=\varphi_{1}(\rho) \quad \text { for all } \rho \in P, \pi \in W .
$$

In particular $X(\pi)=\varphi_{1}(1)=X(1)$ for all $\pi \in W$. Let $V$ denote the kernel of $X$; then $V$ is a normal subgroup $G$ and $W \subset V$. Suppose that $W \neq\{1\}$. Then it follows from the assumption that $G$ has no nontrivial subgroups of order prime to $p$ and from Proposition 2.2.i that $P \subset V$, in contradiction to the fact that $X(\sigma)=-1$ for $\sigma \in P^{\sharp}$. Consequently $W=\{1\}, P$ contains the centralizer of each of its nonunit elements and by Theorem 2 of [2] $G$ is either of type (B), or of type $C$, or $G \cong P S L\left(2, p^{a}-1\right)$, where $a>1$ and $p^{a}-1=2^{b}$. In view of Lemma 3.1 of [3], the only solution of the above equation with $a>1$ is: $p=3, a=2$ and $b=3$. Thus if $a>1, G \cong P S L(2,8)$. Since the groups of types (B), (C) and (D) satisfy the conditions of Theorem 2, the proof is complete.

4. Proof of Theorem $1^{*}$. It follows from Theorem 2 that one of the statements (B), (C) and (D) holds. Statement (A) could not occur, since then

$$
n \geqq\left(p^{a}+3\right) / 2, \quad o(G) \geqq 2 p^{a}\left(p^{a}+3\right) p^{a} / 2>p^{3 a}
$$

a contradiction.

In cases (C) and (D) $o\left(G^{*}\right)>p^{3 a} / 2$ and therefore $G \cong G^{*}$, yielding statements (II)* or (IV)*. In case (B), $o\left(G^{*}\right)=\left(p^{3 a}-p^{a}\right) / 2$ and therefore either $\left[G: G_{0}\right]=2, G_{0} \cong G^{*}$, or $G=G_{0}, o(M)=1$ or 2 . If $\left[G: G_{0}\right]=$ 2 , then $o(M)=1, G$ is isomorphic to a subgroup of the automorphism group of $P S L(2, \mathrm{p})$ and by [5, Lemma 2] $G \cong P G L(2, p)$, yielding $(V)^{*}$. If $G=G_{0}$ and $o(M)=1$, then $G \cong P S L(2, p), p>3$, and $(I)^{*}$ holds. Finally, if $G=G_{0}$ and $o(M)=2$, then it follows from a theorem of 
Schur [4, p. 120] that $G$ is either isomorphic to $S L(2, p)$ and (III)* holds, or it is isomorphic to $\operatorname{PSL}(2, p) \times M$ and (VI)* holds. Since the groups mentioned in Theorem $1^{*}$ satisfy the conditions of that theorem, the proof is complete.

\section{REFERENCES}

1. R. Brauer, and W. F., Reynolds, On a problem of E. Artin, Ann. of Math. 68 (1958), 713-720.

2. M. Herzog, On finite groups containing a CCT-subgroup with a cyclic Sylow subgroup, Pacific J. Math. 25 (1968), 523-531.

3. - On finite groups with cyclic Sylow subgroups for all odd primes, Israel J. Math. 6 (1968), 206-216.

4. I. Schur, Untersuchungen über die Darstellung der endlichen Gruppen durch gebrochene lineare Substitutionen, Crelle J. 132 (1907), 85-137.

5. M. Suzuki, On finite groups with cyclic Sylor subgroups for all odd primes, Amer. J. Math. 77 (1955), 657-691.

6. - Finite groups of even order in which Sylow 2-groups are independent, Ann. of Math. 80 (1964), 58-77.

Received May 10, 1968. This research was supported by the National Science Foundation under Grant NSF-GP-8968.

University of California, SANTA Barbara 



\section{PACIFIC JOURNAL OF MATHEMATICS}

\section{EDITORS}

H. ROYDEN
Stanford University
Stanford, California

\section{R. R. PHELPS}

University of Washington

Seattle, Washington 98105
J. DUGUNDJI

Department of Mathematics

University of Southern California

Los Angeles, California 90007

\section{RICHARD ARENS}

University of California

Los Angeles, California 90024

\section{ASSOCIATE EDITORS}

E. F. BeCKenbaCh
B. H. NEUMANN

F. WOLF
K. YoshidA

\section{SUPPORTING INSTITUTIONS}

\author{
UNIVERSITY OF BRITISH COLUMBIA \\ CALIFORNIA INSTITUTE OF TECHNOLOGY \\ UNIVERSITY OF CALIFORNIA \\ MONTANA STATE UNIVERSITY \\ UNIVERSITY OF NEVADA \\ NEW MEXICO STATE UNIVERSITY \\ OREGON STATE UNIVERSITY \\ UNIVERSITY OF OREGON \\ OSAKA UNIVERSITY \\ UNIVERSITY OF SOUTHERN CALIFORNIA
}

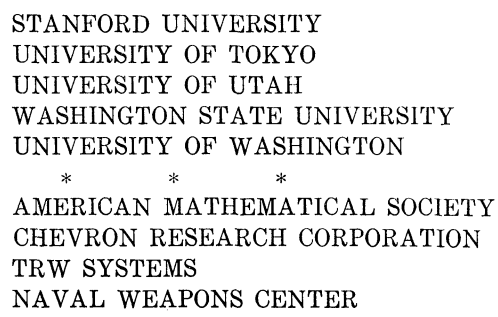

The Supporting Institutions listed above contribute to the cost of publication of this Journal, but they are not owners or publishers and have no responsibility for its content or policies.

Mathematical papers intended for publication in the Pacific Journal of Mathematics should be in typed form or offset-reproduced, double spaced with large margins. Underline Greek letters in red, German in green, and script in blue. The first paragraph or two must be capable of being used separately as a synopsis of the entire paper. It should not contain references to the bibliography. Manuscripts, in duplicate if possible, may be sent to any one of the four editors. Please classify according to the scheme of Math. Rev. 36, 1539-1546. All other communications to the editors should be addressed to the managing editor, Richard Arens, University of California, Los Angeles, California, 90024.

50 reprints are provided free for each article; additional copies may be obtained at cost in multiples of 50 .

The Pacific Journal of Mathematics is published monthly. Effective with Volume 16 the price per volume (3 numbers) is $\$ 8.00$; single issues, $\$ 3.00$. Special price for current issues to individual faculty members of supporting institutions and to individual members of the American Mathematical Society: $\$ 4.00$ per volume; single issues $\$ 1.50$. Back numbers are available.

Subscriptions, orders for back numbers, and changes of address should be sent to Pacific Journal of Mathematics, 103 Highland Boulevard, Berkeley, California, 94708.

PUBLISHED BY PACIFIC JOURNAL OF MATHEMATICS, A NON-PROFIT CORPORATION

Printed at Kokusai Bunken Insatsusha (International Academic Printing Co., Ltd.), 7-17, Fujimi 2-chome, Chiyoda-ku, Tokyo, Japan. 


\section{Pacific Journal of Mathematics \\ Vol. 29, No. $2 \quad$ June, 1969}

Bruce Langworthy Chalmers, On boundary behavior of the Bergman kernel function and related domain functionals ................... 243

William Eugene Coppage, Peirce decomposition in simple Lie-admissible power-associative rings .............................. 251

Edwin Duda, Compactness of mappings...................... 259

Earl F. Ecklund Jr., On prime divisors of the binomial coefficient......... 267

Don E. Edmondson, A modular topological lattice ............... 271

Phillip Alan Griffith, A note on a theorem of Hill ................... 279

Marcel Herzog, On finite groups with independent cyclic Sylow

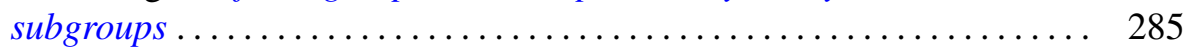

James A. Huckaba, Extensions of pseudo-valuations................. 295

S. A. Huq, Semivarieties and subfunctors of the identity functor ........ 303

I. Martin (Irving) Isaacs and Donald Steven Passman, Finite groups with small character degrees and large prime divisors. II ............ 311

Carl Kallina, A Green's function approach to perturbations of periodic

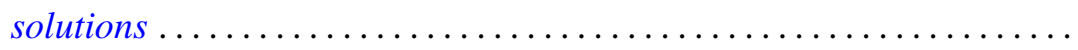

$\mathrm{Al}$ (Allen Frederick) Kelley, Jr., Analytic two-dimensional subcenter manifolds for systems with an integral ....................

Alistair H. Lachlan, Initial segments of one-one degrees ............ 351

Marion-Josephine Lim, Rank k Grassmann products ............. 367

Raymond J. McGivney and William Henry Ruckle, Multiplier algebras of

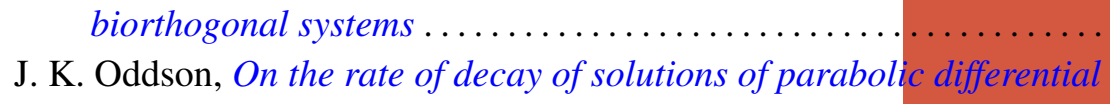

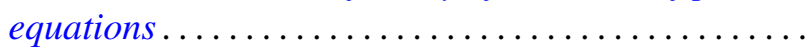

Helmut R. Salzmann, Geometries on surfaces ........... .

Annemarie Schlette, Artinian, almost abelian groups and their groups of automorphisms ............................

Edgar Lee Stout, Additional results on modules over polydisc algebras ...

Lajos Tamássy, A characteristic property of the sphere . .

Mark Lawrence Teply, Some aspects of Goldie's torsion theory. ...

Freddie Eugene Tidmore, Extremal structure of star-shaped sets ...

461

Leon Jarome Weill, Unconditional and shrinking bases in locally convex spaces... 